Editorial

\title{
El aprendizaje pleno del proceso Salud Enfermedad Atención. Reflexiones y adecuaciones didácticas para los primeros años de carreras en ciencias de la salud.
}

\section{Humberto Jure; Maria Beatriz Ciuffolini; Patricia Demuth}

En su libro "El Aprendizaje Pleno-Principios de la Enseñanza para Transformar la Educación", David Perkins (2010) con un enfoque innovador y transformador, nos propone implementar estrategias pedagógicas significativas que permitan hacer de los espacios educativos ámbitos atractivos y productivos para los estudiantes y los docentes. El autor utiliza el juego de béisbol como metáfora para ilustrar su teoría del aprendizaje a la que denomina "Aprendizaje Pleno". Destaca que esa misma metáfora podía construirse, con cualquier otra habilidad o actividad que aprendiésemos a realizar de manera más o menos correcta (tocar un instrumento, jugar al fútbol, aprender un idioma, etc); en ejemplo para adecuaciones didácticas, explica que él aprendió a jugar al béisbol de pequeño con la ayuda de su padre y que lo fue haciendo de una manera informal y progresiva. A partir de esta experiencia, pudo delinear los siete principios del aprendizaje que señalamos a continuación:

1. Jugar el juego completo

2. Lograr que valga la pena el juego

3. Trabajar sobre las partes difíciles

4. Jugar de visitante

5. Descubrir el juego oculto

6. Aprender del equipo y de los otros equipos

7. Aprender el juego del aprendizaje

Si enfocamos esta propuesta en nuestro campo de estudio (el campo de las ciencias de la salud), realizar un Abordaje Integral del Proceso Salud Enfermedad Atención (jugar el juego completo), requiere asumir el desafío de reflexionar sobre nuestros espacios de práctica (lograr que valga la pena el juego), y comprender que nuestras formas de pensar y organizar el proceso de enseñanza- aprendizaje (trabajar sobre las partes difíciles), presupone cierta manera de conceptualizar el problema de saludenfermedad, en cierto tiempo y en cierto contexto familiar y comunitario (jugar de visitante). Debemos considerar que los modos de construcción y reconstrucción de los aprendizajes se encuentran indefectiblemente vinculados a ciertos intereses (descubrir el juego oculto) que sustentan las prácticas de los equipos interdisciplinarios (aprender del equipo) y que permiten el análisis y reflexión crítica sobre el proceso enseñanza -aprendizaje en las facultades de ciencias de la salud (aprender el juego del aprendizaje).

Esta propuesta requiere pensar desde el primer momento en "jugar el juego completo" con nuestros estudiantes de diferentes carreras y definir desde el inicio que entendemos por salud y enfermedad, y cual es nuestro objeto/sujeto de estudio. En este marco debemos considerar que el modo con el que pretendemos dar respuesta a un problema de salud está íntimamente relacionado a la forma de interpretación, 
análisis y comprensión del mismo. Esta perspectiva se traduce en la práctica, en el ejercicio de ciertas formas de cuidado de la salud, las cuales incluyen desde acciones de autocuidado, pasando por el ejercicio de ciertas habilidades y destrezas profesionales, que finalmente se traducen en determinados modelos de estructuración y organización de los servicios de salud.

En nuestras facultades de ciencias de la salud la mayoría de los profesores fragmentan los aspectos que quieren enseñar en elementos simples, procurando la clasificación taxonómica de los mismos. Así es como un proceso complejo se descompone en sus elementos más pequeños, hasta tal punto que se convierten en fragmentos sin sentido y se pierde de vista el todo. Esta división y fragmentación en partes es una de las características estructurales del modelo biomédico de concepción biologista y de orientación curativa que no considera el contexto, ni la historia. Un enfoque que se identifica con la racionalidad científica, la separación entre teoría y práctica, la medicalización de los problemas y la comprensión de la salud- enfermedad como una mercancía (Menéndez, 2004). A esta distorsión en la perspectiva, Perkins la denomina elementitis y según su concepción se une estrechamente al empeño en enseñar información sobre las cosas, en lugar de las cosas mismas, aspecto al que denomina informatitis.

En este sentido, existe en nuestras facultades, una permanente tensión entre una concepción holística del proceso salud- enfermedad, que intenta comprender a la persona y su contexto y una concepto reduccionista y determinista (elementitisinformatitis) que entiende a la enfermedad como un fenómeno específico que cobra entidad en si mismo y se estructura alrededor del creciente desarrollo de la aparatología y la industria del medicamento, teniendo como eje el diagnóstico y tratamiento de la enfermedad.

Desde la perspectiva del Aprendizaje Pleno, se promueve una visión integral, es decir, si tras una sesión de práctica de fútbol, en la que trabajásemos el mejoramiento técnico de cómo se realiza un pase, jugáramos un partido de fútbol de verdad, entonces esa práctica aislada cobraría sentido, y sería incluso necesaria. A esta concepción, Perkins (2010), la denomina en sus siete principios "jugar el partido completo". Este aspecto, resulta fundamental en todo aprendizaje para no perder el sentido de lo que se está haciendo, y para que se cumpla el principio de que "valga la pena jugar". Este sería el segundo principio y se relaciona directamente con la motivación intrínseca que se tiene sobre el objeto/sujeto de estudio, en nuestro caso la persona en su contexto familiar y comunitario.

El tercer principio se refiere a "trabajar sobre las partes difíciles", bajo esta premisa, deberíamos aprovechar al máximo la motivación en los comienzos del proceso de enseñanza- aprendizaje en nuestras instituciones educativas, estimulando la comprensión, considerando las expectativas del estudiante, los desafíos planteados y su capacidad de crear y sintetizar. Perkins (2010) afirma que no se trata meramente de practicar las partes difíciles en el sentido de repetirlas, sino que implica deconstruirlas y reconstruirlas de modo que se ejecuten de una forma distinta. Morin (2005) plantea la necesidad de realizar una doble operación de análisis a los fines de hacer inteligible la complejidad del proceso salud-enfermedad, lo que requiere por una parte procesos operacionales que permitan jerarquizar, distinguir, seleccionar y ordenar los 
elementos constitutivos y que por otra parte faciliten religar, asociar dichos componentes, de manera tal de poder "distinguir sin desarticular y asociar sin reducir". Sin embargo, el modelo tradicional de enseñanza de la medicina reafirma el desarrollo de una propuesta hospitalocéntrica, biologicista y tecnocrática, con procesos de enseñanza centrados en el docente y la transmisión de conocimientos fraccionados y descontextualizados.

El desarrollo del cuarto principio: "Jugar de visitante", implica lo contrario, es decir, contextualizar el aprendizaje. Significa transferir el aprendizaje aplicando conocimientos teóricos a una situación práctica real y en salud, considerar como unidad funcional de cuidado y abordaje a la persona en su contexto familiar y comunitario. En la constitución de esta perspectiva ha contribuido especialmente la Teoría General de Sistemas al facilitar la comprensión de la importancia de la familia y proporcionado una variedad de herramientas que facilitan el abordaje de demandas y conflictos que emergen de las condiciones de salud de cada miembro en particular y de la familia en general. Otro aporte en este sentido lo constituye la propuesta de lan Mc Whinney (2003) denominada Proceso Clínico Centrado en el Paciente, que consiste en comprender el proceso salud enfermedad desde una perspectiva integral $y$ considera en la atención los aspectos biológicos, emocionales, y contextuales junto a las expectativas de las personas, valorando además la interacción humana y ampliando la capacidad comprensiva del método clínico.

El quinto principio, "descubrir el juego oculto", considera que casi todo lo que aprendemos tiene sus aspectos ocultos, dimensiones y perspectivas que no son evidentes en la superficie de la actividad. En el caso específico de salud, un abandono de la idea de método en función de la noción de proceso, y el corrimiento del objeto/sujeto de cuidado desde el individuo enfermo hacia la persona en su contexto familiar y comunitario, constituye sin dudas el punto de ruptura con el paradigma dominante y abre la posibilidad de una perspectiva crítica respecto a aquellos intereses económicos dominantes que se vinculan entre si y orientan las decisiones sistémicas en el contexto global.

El sexto principio, "aprender del equipo", parte del supuesto de que el aprendizaje cotidiano concibe la construcción del conocimiento, la habilidad y la comprensión, como un emprendimiento colectivo, por lo que se considera prudente aprender del equipo y de los demás equipos que participan en el proceso enseñanza aprendizaje, espacio donde invariablemente se producen múltiples interacciones. El equipo es ese conjunto de los seres interrelacionados en el que cada persona posee su singularidad, como ser extremadamente complejo y creativo e interviene en el ritmo de la naturaleza, superando el antropocentrismo histórico, como ser solidario, complementario y creador.

En salud debiéramos asumir el desafío de diseñar una propuesta que integre a los estudiantes de diferentes carreras en una práctica con distintos elementos en común, una nueva práctica, que al mismo tiempo que los prepare para el trabajo en el equipo, permita introducir el enfoque interdisciplinario que facilite una mayor fuerza explicativa al abordaje de los problemas sociosanitarios y ayude a sus integrantes, a actuar más allá de la lógica de su propio interés y en favor del interés general, en especial de las personas más vulnerables. 
El séptimo y último principio es el de "aprender el juego del aprendizaje", se vincula a qué aspectos pueden aprenderse acerca del juego del aprendizaje y cómo van a aprenderse los mismos. Se ha demostrado en diferentes estudios, la influencia en los estudiantes de experiencias educativas vinculadas a la atención primaria de la salud y al trabajo comunitario, actividades que han permitido mejorar las actitudes, el respeto y la empatía hacia las personas, aprender acerca de los roles y responsabilidades profesionales, conocer los sistemas sanitarios y las necesidades de salud de una población y en especial como estímulo para el desarrollo de autoconfianza y una perspectiva ética en el ejercicio de la profesión. Además se constituyen en experiencias movilizadoras que permiten ahondar en los procesos individuales de generación de los conocimientos y en las concepciones con los que se opera sobre la realidad. Es por eso que este principio estimula la búsqueda de estrategias que permitan hacer visible los pensamientos y razonamientos más profundos vinculados a la autorregulación de los propios conocimientos y competencias. Desde los primeros años y respetando la versión para principiantes seleccionada se buscaría que los estudiantes externalicen esos procesos, profundicen en su comprensión y desarrollen un mayor dominio sobre el propio aprendizaje. Desde esta mirada, se entiende que comprender los propios procesos de cognición genera un movimiento que les, y nos (profesores), permitirá visualizar los procesos de aplicación, análisis y evaluación que han venido realizando en diferentes momentos del cursado de las carreras.

El principal problema que encontramos los docentes para enseñar a nuestros alumnos situaciones problemáticas de cierta profundidad, es su abordaje inicial. Perkins (2010) señala que no hay ninguna necesidad de comenzar con un emprendimiento a escala completa para utilizar los Siete Principios del Aprendizaje Pleno. Propone como modelo de todo aprendizaje la "versión para principiantes". Esta propuesta se refiere a una práctica que ponga a los alumnos en contacto directo con los problemas de salud de una comunidad y contribuya a desarrollar aquellas habilidades necesarias para definirlos, abordarlos y comenzar a resolverlos. Del mismo modo que el autor jugaba béisbol, con menos integrantes en los equipos, menos bases y menos reglas, debemos buscar "versiones en pequeño" de aquellos saberes, procedimientos y habilidades que queremos que nuestros alumnos adquieran.

Si quisiéramos trasladar esta propuesta de Aprendizaje Pleno a nuestro ámbito educativo y debatir sobre modelos de enseñanza-aprendizaje, debiéramos en primer lugar considerar que los mismos se inscriben en un marco de referencia mayor que refiere tanto a cuestiones de orden estrictamente pedagógico, como también a las condiciones de contexto socio-histórico. En este marco se propone desde el inicio de las carreras un desplazamiento del espacio áulico hacia los escenarios reales, donde se procurará la adquisición de competencias que estimulen un abordaje integral y que faciliten la articulación teórico-práctica, asumiendo la necesidad de un compromiso ético y social. Nuestra propuesta, se basa en propiciar en los alumnos un contacto directo con los problemas de salud de una comunidad en un tiempo y lugar determinados. Y estimular en el educando una perspectiva epidemiológica, que como ciencia crítica, contribuya a desarrollar aquellas habilidades necesarias para definir dichos problemas, abordarlos y comenzar a resolverlos, promoviendo los modos de vida sustentables y saludables. Se propone una sistematización de las características esenciales de un sistema de contradicciones entre procesos protectores y procesos 
deteriorantes y la identificación de tales procesos como un insumo para la acción, ya sea que evite y atenúe los procesos deteriorantes (estrategias de prevención) o fortalezca y desarrolle los procesos protectores (estrategias de promoción de la salud) (Breilh, 2003).

Para asumir este desafío de nuevas prácticas, no basta el conocimiento, necesitamos una nueva mente y un nuevo corazón.

\section{BIBLIOGRAFIA}

1. Perkins David. El Aprendizaje Pleno. Principios de la enseñanza para transformar la educación. Buenos Aires. Editorial Paidos. 2010

2. Breilh, Jaime. Epidemiología Crítica. Ciencia Emancipadora e Interculturalidad. Buenos Aires. Editorial Lugar. 2003

3. Ciuffolini MB, Jure H. "Estrategias de comprensión integral del proceso salud- enfermedad: aportes desde la perspectiva de la vivienda saludable". Astrolabio. Centro de Estudios Avanzados. UNC. № 3. Noviembre 2006.

4. Mc Whinney Ian. Medicina de Familia. Madrid. Editorial Doyma.1995

5. Menendez Eduardo. "Modelos de atención de los padecimientos: de exclusiones teóricas y articulaciones prácticas". Salud Colectiva. Spinelli Hugo, Compilador. Buenos Aires: Editorial Lugar. 2005

6. Morin, Edgar. Introducción al pensamiento Complejo. Editorial Gedisa. Barcelona.2005 